
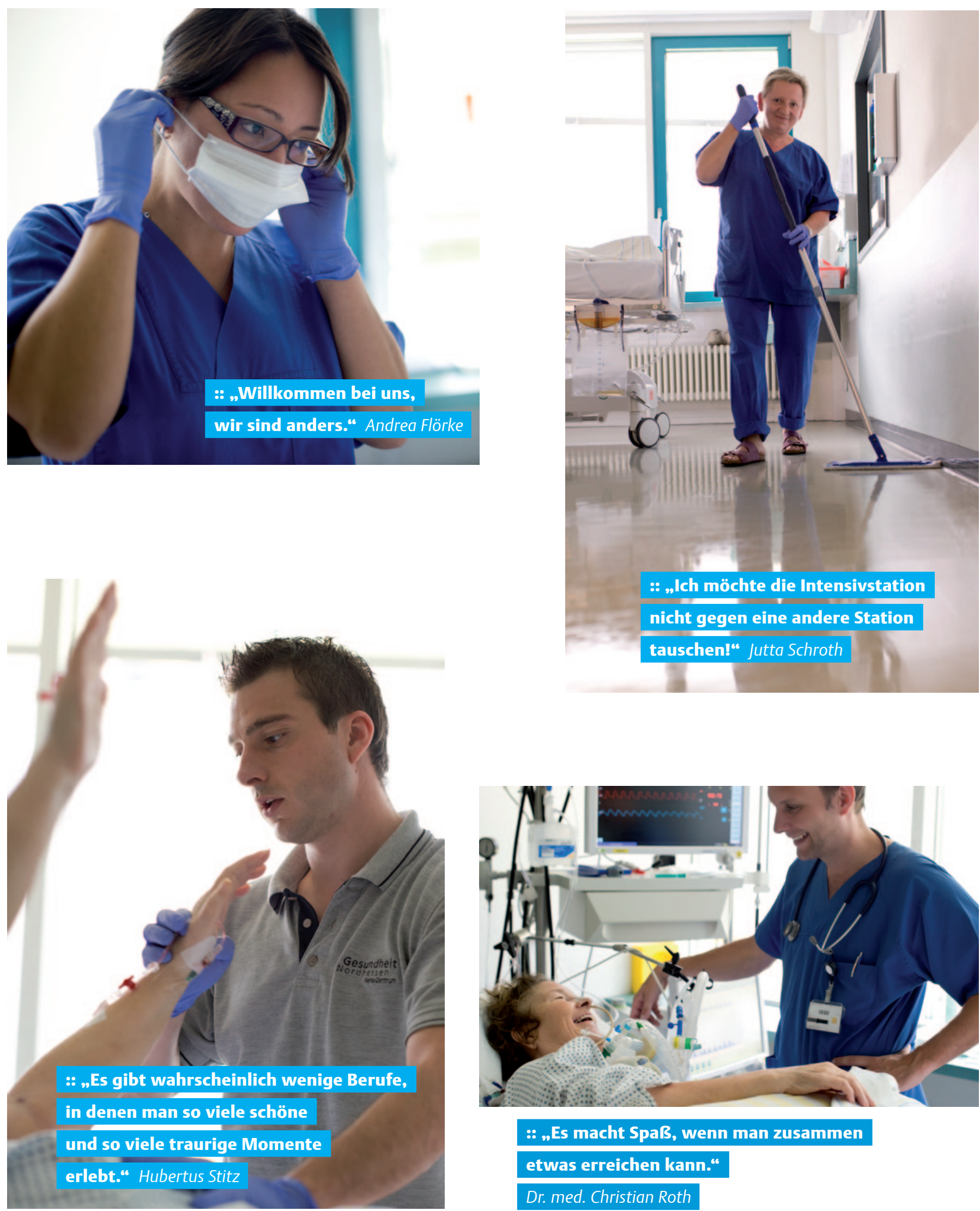

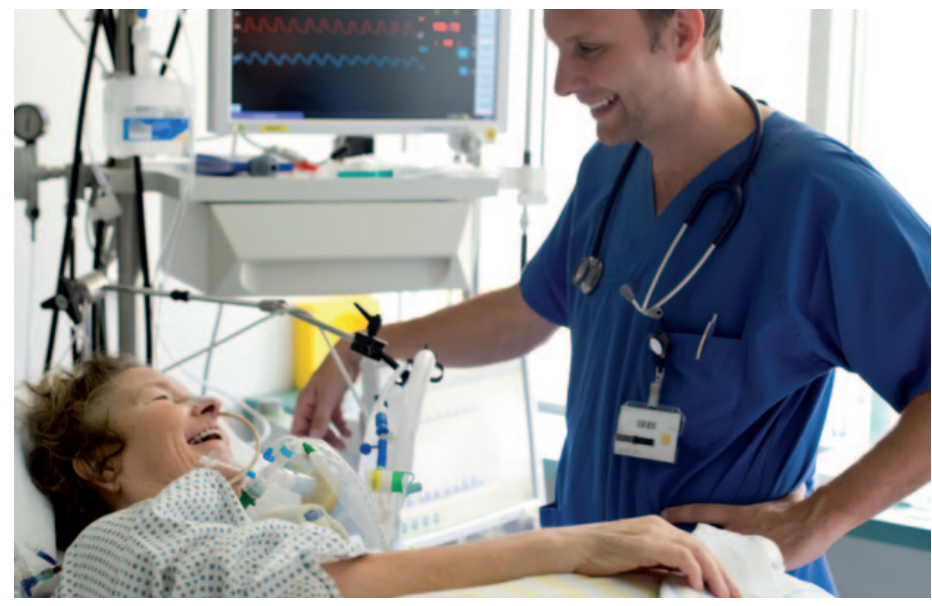

:: „Es macht Spaß, wenn man zusammen

etwas erreichen kann.“

Dr. med. Christian Roth 


\section{Pflege mit anderen Augen}

REPORTAGE Haben Sie sich schon einmal gefragt, was die Reinigungskraft, der Sie fast täglich begegnen, über Ihren Beruf denkt? Oder können Sie sich vorstellen, wie die angrenzenden Berufsgruppen, mit denen Sie täglich zu tun haben, die Intensivpflege sehen? Wir haben eine Reinigungskraft, einen Physiotherapeuten und einen Intensivmediziner zu ihrem persönlichen Blick auf die Intensivpflege befragt. In dieser Reihe darf natürlich die Intensivpflegekraft nicht fehlen, die erzählt, wie sie ihren eigenen Beruf sieht.

Alle Interviewten arbeiten auf der neurologisch/ neurochirurgischen Intensivstation des Klinikums Kassel, das mit 28 Fachkliniken und Instituten das größte kommunale Krankenhaus in Hessen ist. Die Klinik verfügt über zwei chirurgische, eine internistische, eine neurologischneurochirurgische und eine neonatologische Intensivstation. Seit Dezember ist das Kinderkrankenhaus Park Schönfeld ebenfalls auf dem Klinikgelände ansässig, sodass nun auch eine pädiatrische Intensivstation dazugehört.

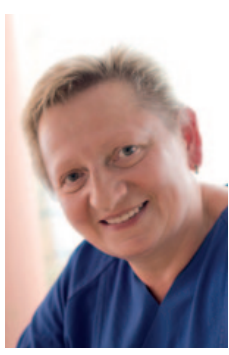

Jutta Schroth (55), gelernte Einzelhandelskauffrau, seit 17 Jahren Reinigungskraft auf der neurologisch/neurochirurgischen und den beiden chirurgischen Intensivstationen

- Wie haben Sie Ihre Anfangszeit auf der Intensivstation erlebt, zwischen den schwer kranken Menschen und den Geräten und Schläuchen?

Am Anfang war alles komisch, da hat man ganz vorsichtig gearbeitet. Es war sehr schwierig, weil man nicht gut um die ganzen Geräte herum putzen konnte. Da muss man sehr aufpassen, dass man nichts umkippt oder abreißt und dabei die strengen Hygienevorschriften erfüllt.

Damals hat es mich schwer belastet, wie krank die Patienten sind und wie oft welche sterben. Heute kann ich damit umgehen, ich denke, ich bin da reingewachsen.

\section{- Wie sind Sie mit dieser Belastung fertig geworden?}

Damit musste ich allein fertig werden. Dann habe ich zu Hause meinem Mann viel erzählt und er hat mir immer zugehört. Aber wenn sehr junge Patienten schwer krank sind oder sterben, nimmt mich das auch heute immer noch sehr mit.

\section{- Welche Berührungspunkte haben Sie mit den Intensivpflegern?} Meine Vorarbeiterin sagt mir, was zu machen ist, meine Arbeit erledige ich selbstständig. Die Intensivpfleger sehe ich zwar, aber ich habe mit ihnen kaum etwas zu tun. Manchmal, wenn etwas dringend ist, bitten sie mich, etwas zuerst zu reinigen.
- Was denken Sie über die Arbeit der Intensivpflegekräfte? Ich denke, das ist ein körperlich und seelisch harter Beruf, in dem man viel unter Stress steht. Sie geben immer ihr Bestes, aber es ist ganz enorm, welcher Belastung sie ausgesetzt sind. Mir ist aufgefallen, dass die Pflegekräfte oft schon sehr jung ganz schön angeschlagen sind - der eine hat Probleme mit den Knien, der andere mit dem Rücken. Außerdem ist die Schichtarbeit heftig.

\section{- Erscheinen Ihnen die Pflegekräfte glücklich?}

Ja, sie sind zumindest immer gut gelaunt. Sie müssen den Patienten ja auch Gutes tun, da muss man immer gute Laune haben.

\section{- Welches Erlebnis ist Ihnen in Erinnerung geblieben?}

Das ist mindestens schon zehn Jahre her, aber daran denke ich sehr oft: Eine schwer kranke ältere Dame lag auf der Station und immer, wenn ich an ihr Bett kam und das Bettgitter geputzt habe, wollte sie nach meiner Hand greifen. Sie tat mir so leid, weil sie so lange da lag und keine Angehörigen hatte, die sie besuchten. Wenn ich in das Zimmer kam, standen mir eigentlich schon die Tränen in den Augen. Aber dann ist die Frau nach einem Jahr im Krankenhaus gesund nach Hause gegangen. Später hat sie uns dann auch noch ein paar Mal besucht, das fand ich ganz toll.

\section{- Würden Sie gern auf einer anderen Station arbeiten?}

Nein, ich möchte die Intensivstationen nicht gegen eine andere Station tauschen. Die Intensivstation ist nicht jedermanns Sache, aber mir macht meine Arbeit viel Spaß. Die Ärzte und Pfleger sind sehr nett. Sie behandeln mich ganz normal, sind immer freundlich und grüßen. Das ist nicht überall so. Ich könnte nicht auf einer Kinderkrebsstation arbeiten, das würde ich emotional nicht aushalten.

\section{- Könnten Sie sich vorstellen, als Intensivpflegerin zu arbeiten?} Ich finde den Beruf sehr interessant, man hat mit vielen Menschen zu tun, mit verschiedenen Krankheiten und wie man versucht, die Patienten zu heilen. Meine Tochter ist Krankenpflegerin, das war immer ihr Wunschberuf, aber ich kann nicht sagen, ob es mein Traumberuf wäre. Ich ziehe auf jeden Fall den Hut davor, was diese Leute leisten. 


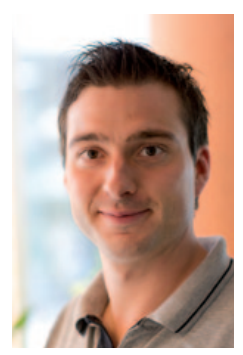

Hubertus Stitz (26), seit 6 Jahren Physiotherapeut auf der Intensivstation

- Was haben Sie gedacht, als sie zum ersten Mal auf die Intensivstation gekommen sind und die ganzen Schläuche und Geräte gesehen haben?

Das erste Mal war ich im Rahmen eines Praktikums in der Ausbildung auf der Intensivstation, da hatte ich schon ein mulmiges Gefühl.

Als ich dann angefangen habe, dort zu arbeiten, war es am Anfang schwierig. Während der Behandlung hatte ich manchmal Angst, aus Versehen ein Kabel oder einen Schlauch rauszuziehen oder den Patienten zu überfordern. Damals habe ich die Geschichten der Patienten auch noch mehr an mich herangelassen und mir auch zu Hause viele Gedanken gemacht.

\section{- Hat Ihre Ausbildung Sie auf die Intensivstation vorbereitet?} Nicht unbedingt. Man spricht mal darüber, wie die Arbeit dort aussehen könnte, aber ich würde nicht sagen, dass man nach der Ausbildung darauf vorbereitet ist. Das ist auch das Schwierige daran, man ist darauf angewiesen, wer einen auf der Intensivstation führt und wer einem Sachen erklärt, sei es vonseiten der Pflege oder der Ärzte. Man muss sich auch selbst viel aneignen und anlesen.

\section{- Ist die Intensivpflege in Ihren Augen ein erfüllender oder trau- riger Beruf? \\ Sowohl als auch. Es gibt wahrscheinlich wenige Berufe, in denen man so viele schöne und so viele traurige Momente erlebt. Gerade wenn Kinder oder junge Menschen versterben oder die Station schwer ge- schädigt verlassen, ist das besonders schlimm. Aber wenn ein Patient um den es anfänglich sehr schlecht stand, nach einem Jahr erzählt, er hat wieder angefangen zu studieren, ist das sehr schön.}

- Was, denken Sie, macht den Reiz aus, diesen Beruf zu ergreifen? Ich glaube, der Reiz liegt darin, dass der Stellenwert innerhalb der Pflege höher angesiedelt ist. Das heißt, dass die Verantwortung, die man dort trägt, größer ist, denn die Patienten sind alle überwachungspflichtig. Außerdem arbeitet die Pflege hier eng mit den Ärzten zusammen, braucht ein sehr gutes medizinisches Hintergrundwissen und kann viele Sachen machen, die die Pflege auf einer normalen Station nicht machen darf.

\section{- Was sind Intensivpfleger für eine Art von Menschen?}

Wenn man die ganze Breite sieht, gibt es alle Typen Mensch, die es im normalen Leben auch gibt. Aber ich denke, wenn man diesen Beruf ergreift, muss man eine gewisse soziale Kompetenz haben und Menschen gern helfen. Außerdem muss man sehr interessiert sein, denn die Anforderungen sind sehr hoch und die Ausbildung dauert genauso lange wie die meisten Hochschulstudiengänge. Einen, der als oberstes Ziel hat, Millionär zu werden, wird man in der Intensivpflege wohl kaum finden.

\section{- Warum haben Sie sich für die Intensivstation entschieden?} Ich arbeite nicht nur auf der Intensivstation, sondern auch auf der Schlaganfallstation. Auf diesen Stationen ist der therapeu- tische Anspruch am höchsten. Gerade in der Neurologie ist es nicht einfach, Funktionen wieder herzustellen. Manchmal dauert es Jahre, bis ein Patient bestimmte Fähigkeiten wieder erlernt, das kann man nicht mit einem gebrochenen Knie vergleichen.

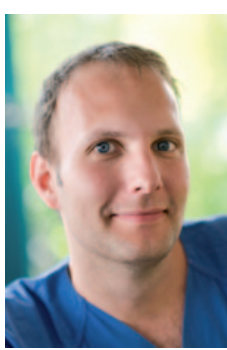

Dr. med. Christian Roth (36), Facharzt für Intensivmedizin, seit zweieinhalb Jahren Oberarzt

- Ist die Intensivpflege in Ihren Augen ein erfüllender Beruf?

Ja, man kann als Intensivpflegekraft viel machen, hat die Möglichkeit, tiefe Einblicke in die Funktion des Menschen zu bekommen, und sieht, dass schwer kranke Patienten geheilt werden können. Auf der anderen Seite muss man aber ganz klar sagen, dass es ein körperlich und emotional anstrengender Beruf ist. Deshalb muss man aufpassen, dass man sich nicht verbraucht und einen guten Ausgleich neben der Arbeit hat.

\section{- Ist dieser Beruf auch gesellschaftlich anerkannt?}

Ja, auf jeden Fall. Das ist auch die Rückmeldung, die wir von den Angehörigen bekommen. Sie sehen, wie viel die Kollegen arbeiten und wie anstrengend diese Arbeit ist.

- Wie sehen Sie Ihre Berührungspunkte mit der Intensivpflege? Die sind sehr eng geknüpft, wir arbeiten zusammen in einem Team und wollen das Gleiche erreichen, nämlich, dass es dem Patienten besser geht. Im ärztlichen Bereich sind wir auf die Informationen von der Pflege angewiesen, weil die natürlich viel mehr Zeit bei dem Patienten verbringt. Umgekehrt brauchen die Pflegekräfte die Informationen von den Ärzten, wie die Planung für die Therapie aussieht und wie es weitergeht. Das ist immer ein Austausch und das kann nur in einem Miteinander funktionieren. Dann macht es Spaß, wenn man zusammen etwas erreichen kann.

- Was, denken Sie, macht den Reiz aus, diesen Beruf zu ergreifen? Der Beruf ist sehr interessant und abwechslungsreich. Die Intensivpflegekräfte sind nicht nur für die Pflege zuständig, sondern auch für die Überwachung oder bestimmte Therapien, sie machen medizinisch gesehen viel mehr als eine Pflegekraft auf einer peripheren Station. Der einzige Haken daran ist, dass es bei knapper werdenden Ressourcen schwieriger wird, eine solche Hightech-Medizin zu betreiben. Deshalb muss man das Gesundheitssystem im Auge haben.

\section{- Warum haben Sie sich für die Intensivmedizin entschieden?} Das hat mich schon immer interessiert, das ist ein Fach, wo immer viel los ist, wo es viel Bewegung gibt, wo man aber auch das Gefühl hat, dem Patienten etwas Gutes zu tun. Nicht nur medizinisch gesehen, sondern auch emotional ist das Spektrum sehr breit. Von der Freude, wenn man den Angehörigen sagen kann, dass es dem Patienten besser geht, bis hin zu dem Zeitpunkt, an dem man den Hirntod feststellen und einsehen muss, dass der Patient verstorben ist. 
- Können Sie sich daran erinnern, wie es war, als Sie als Student das erste Mal auf eine Intensivstation kamen?

Man hat als Student den Vorteil, dass man schon mal gehört hat, wie es auf der Intensivstation abläuft. Wobei es dann doch noch mal anders war, als ich zum ersten Mal auf eine Intensivstation gekommen bin. Als ich in diesen abgeschlossenen Bereich kam, hat mich erst einmal ein Gefühl von Unbehagen befallen, weil man sich nicht auskennt und nicht genau weiß, was los ist. Je mehr man aber in diesem Alltag lebt, umso selbstverständlicher wird es. Wir dürfen aber nie vergessen, dass das nicht „normal“ ist. Gerade wenn Angehörige zum ersten Mal da sind, muss man sich vor Augen führen, dass dieses Umfeld für andere Menschen beängstigend sein kann.

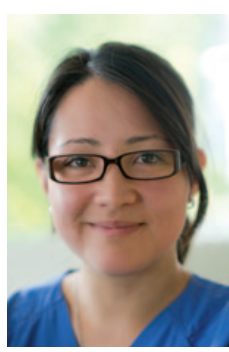

Andrea Flörke (30), seit eineinhalb Jahren Intensivpflegerin

- Warum haben Sie sich für diesen Beruf entschieden?

Ich wollte schon immer im sozialen Bereich und mit Menschen zusammen arbeiten. Zunächst war ich einige Jahre in einer Rechtsanwaltskanzlei tätig, bevor ich mich für die Ausbildung als Krankenpflegerin entschieden habe. Ich wollte von vornherein auf die Intensivstation, weil ich viel lernen und sehen wollte.

\section{- Was gefällt Ihnen besonders gut an Ihrem Beruf?}

Den Menschen zu helfen, wenn es ihnen schlecht geht, und Gutes zu tun. Wenn es den Menschen, die in lebensbedrohlichen Situationen waren, wieder besser geht und man ihnen helfen konnte, ist das sehr schön. Jeder Tag auf der Intensivstation ist anders und die Arbeit ist sehr umfangreich. Man muss körperlich und geistig immer fit sein. Obwohl die Arbeit anstrengend ist, macht sie mir viel Spaß.

\section{- Was ist für Sie schwierig in Ihrem Beruf?}

Ich habe selbst ein Kind, und wenn Kinder oder Jugendliche plötzlich aus dem Leben gerissen werden und bei uns auf Station liegen, ist das schwierig für mich. Mann kann sich das vorher gar nicht vorstellen, wie viele schlimme Sachen auch jungen Menschen passieren. Auf der neurologisch/neurochirurgischen Intensivstation kommt hinzu, dass viele Patienten ihr Leben nicht wieder so aufnehmen können, wie sie es vorher gelebt haben. Diese Patienten nehmen die Erkrankung und den Krankenhausaufenthalt nicht als Abschnitt mit nach Hause, sondern körperliche oder geistige Schäden begleiten sie weiterhin. Das ist oft tarurig.

\section{- Welche Probleme sehen Sie in Ihrem Beruf?}

Auch wir haben zu wenig Personal und gerade auf der Intensivstation ist es wichtig, dass die Patienten gut betreut werden. Man kann das bis zu einem bestimmten Punkt kompensieren, aber irgendwann geht es nicht mehr.

\section{- Ist Ihr Beruf in der Gesellschaft anerkannt?}

Ja, ich glaube schon. Wenn ich jemandem erzähle, was ich beruflich mache, höre ich von den meisten Leuten, dass sie diesen

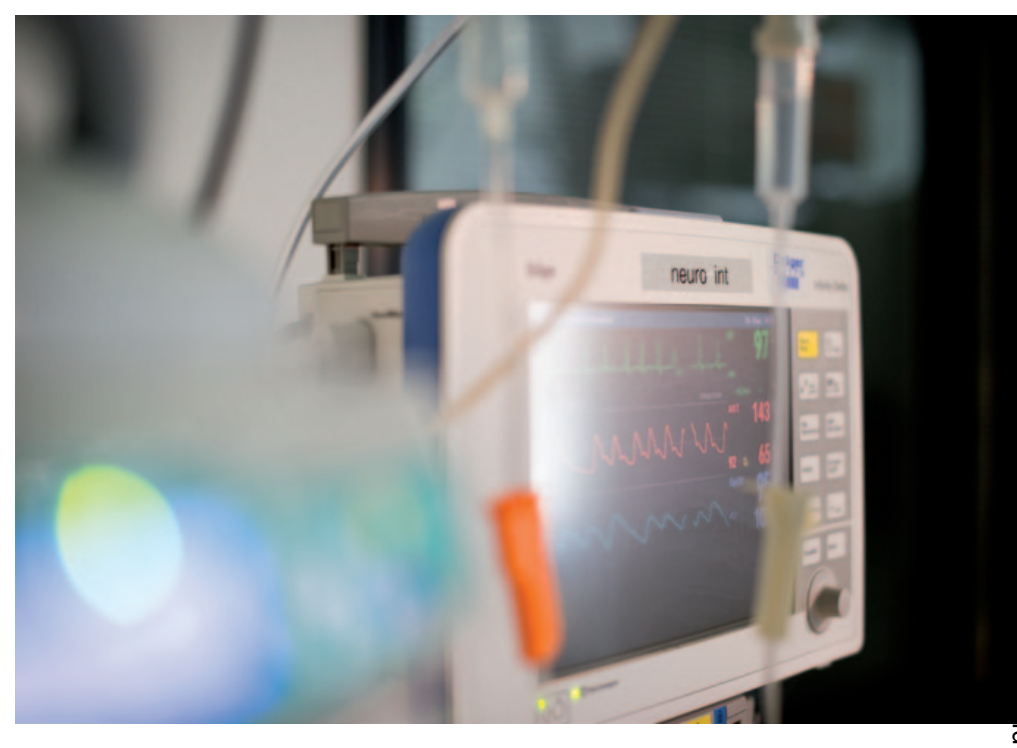

Beruf nicht ausüben könnten und wie schön es sei, dass es Menschen gibt, die das machen.

\section{- Was sind die Intensivpfleger in Ihren Augen für die angren- zenden Berufsgruppen?}

Da wir am meisten Zeit mit dem Patienten verbringen, sind wir eine Art Schnittstelle. Bei uns läuft alles zusammen und wir geben die Informationen an Ärzte, Physiotherapeuten, Ergotherapeuten oder Logopäden weiter, bekommen von ihnen Informationen zurück und organisieren es so, dass die Abläufe funktionieren.

\section{- Welche Art von Menschen sind die Intensivpflegekräfte?}

Viele sagen, dass Intensivpfleger anders wären. So wurde es mir auch am Anfang gesagt: „Willkommen bei uns, wir sind anders.“ Ich kann das noch nicht sagen, ob wir anders sind, ich bin ja noch nicht lange dabei. Ich glaube, dass der Humor trockener ist, weil damit vielleicht einiges verarbeitet wird. Aber sonst sind wir ganz normal, denke ich. Außerhalb des Krankenhauses erkennt uns wahrscheinlich keiner als Intensivpfleger.

\section{AUTORIN}

Mona Herz

Journalistin und Medizinstudentin

Freie Autorin des Georg Thieme Verlags seit 2008 mona.herz@thieme.de

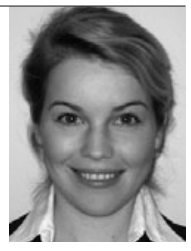

\section{BIBLIOGRAFIE}

DOI 10.1055/s-0031-1298121

Intensiv 2012; 20 (1): 8-11

(c) Georg Thieme Verlag KG

Stuttgart · New York · ISSN 0942-6035 